\title{
A New Approach for Fractional Order Derivative and Its Applications
}

\author{
Ali Karci \\ Department of Computer Engineering, Faculty of Engineering, İnönü University, 44280 Malatya, Turkey \\ *Corresponding Author: adresverme@gmail.com
}

Copyright (C) 2013 Horizon Research Publishing All rights reserved.

\begin{abstract}
The fractional order derivative (FOD) concept is an important concept, since FOD has application area in engineering and science. The concept of FOD can be found in extensive range of many different subject areas. For this reason, the concept of FOD should be examined in detail. After giving different methods mostly used in engineering and scientific applications, the deficiencies, omissions or errors of these methods will be discussed in this study. Some of these methods are Euler, Riemann-Liouville and Caputo which are FOD methods. There are important deficiencies of Euler, Riemann-Liouville and Caputo methods, and these deficiencies were illustrated for constant and identity functions. Due to these deficiencies, FOD fconcept was redefined in this paper. After defining the FOD concept, the applications of FOD for polynomial, exponential, trigonometric and logarithmic functions were handled in this study. Euler, Riemman-Liouville and Caputo methods can be regarded as curve fitting or curve approximation methods not FOD methods. The method in this paper is a new point of view for FOD.
\end{abstract}

Keywords Derivative, Fractional Order Derivatives, Curve Fitting

\section{Introduction}

The FOD methods in the literature can be considered as different approaches instead of classical derivatives. There are a lot of studies in this area and the most of these studies have used Euler, Riemann-Liouville and Caputo FODs. Due to this case, this study focused on Euler, Riemann-Liouville and Caputo FODs.

Some of studies on the FODs can be summarized as follows.

A minimization problem with a Lagrangian that depends on the left Riemann-Liouville FOD was considered in [1] such as finite differences, as a subclass of direct methods in the calculus of variations, consist in discretizing the objective functional using appropriate approximations for derivatives that appear in the problem. There is a study on fractional extensions of the classical Jacobi polynomials [2], and fractional order Rodrigues' type representation formula. By means of the Riemann-Liouville operator of fractional calculus, new g-Jacobi functions were defined, some of their properties were given and compared with the corresponding properties of the classical Jacobi polynomials [2]. There is another study on the discussion of theory of fractional powers of operators on an arbitrary Frechet space, and the authors of this study obtained multivariable fractional integrals and derivatives defined on certain space of test functions and generalized functions [3].

Differential equations of fractional order appear in many applications in physics, chemistry and engineering [4]. There is a requirement for an effective and easy-to-use method for solving such equations. Bataineh et al used series solutions of the fractional differential equations using the homotopy analysis method [4]. Many recently developed models in areas like viscoelasticity, electrochemistry, diffusion processes, etc. are formulated in terms of derivatives (and integrals) of fractional (non-integer) order [5]. There is a collection of numerical algorithms for the solution of the various problems arising in derivatives of fractional order [5].

The fractional calculus is used to model various different phenomena in nature but due to the non-local property of the FODs, it still remains a lot of improvements in the present numerical approaches [6]. There are some approaches based on piecewise interpolation for fractional calculus, and some improvement based on the Simpson method for the fractional differential equations [6].

Recently, many models are formulated in terms of fractional derivatives, such as in control processing, viscoelasticity, signal processing, and anomalous diffusion, and authors of this paper studied the important properties of theRiemann-Liouville derivative, one of mostly used fractional derivatives [7]. The philosophy of integer order sliding mode control is valid also for the systems represented by fractional order operators [8].The FOD methods given in this paper are small part of studies in the literature. There are studies on FODs such as Fractional Order Systems, Fractional Differential Equations, Fractional Integrals, Fractional Numerical Methods, etc. Due to this case, there is 
a need for formal definition for FOD.

The formal definition for FOD was given in [9]. This definition is short and there are not any applications of FODs in [9]. The methods in the literature can be regarded as curve fitting or curve approximation methods. These methods have some errors or deficiencies, and the reason or source of these errors and deficiencies can be summarized as follows.

1) It was assumed that the order of derivative is integer up to a specific step of the methods in the literature. While derivation reached to that step, the order of derivative was converted in to real number. This process concluded in involvement of gamma functions due to the assumption of order of derivative as integer. This is a deficiencies and error. If this case assumed for real values, the obtained coefficient will not be a gamma function, or the gamma function will need new definition.

2) Another important point is that powers of variables decreased as integer during derivation process. There was another assumption in FOD regarded the powers of variables. The coefficients of variables were determined with gamma functions, and powers declining of variables were also regarded as integer up to a specific step, and after reaching that step, power decreasing was assumed as real number. This is not the case in real value powers and coefficients.

In this study, it was focused on the shortcomings and wrong points involved in the methods of Euler, Riemann-Liouville and Caputo for FODs. Especially, the FODs of constant and identity functions will be obtained for Euler, Riemann-Liouville and Caputo methods. Euler and Riemann-Liouville methods were yielded shortcomings and errors in results for constant functions, and on contrary, Caputo method was yielded in correct result for constant function. All methods have not provided accurate results for identity function.

\section{Deficiencies in Euler, Riemman-Liouville and Caputo Methods for FODs}

There are different methods and approximations for FODs since 1730 . There is a common property related to these methods and approximations. The order of derivative as integer caused gamma function involvement in most of the methods and approximations. We used three most popular methods in this study for illustration of deficiencies in the FOD methods.

L.Euler (1730) method:

$$
\frac{d^{n} x^{m}}{d x^{n}}=\frac{\Gamma(m+1)}{\Gamma(m-n+1)} x^{m-n}
$$

where $\Gamma(\mathrm{m}+1)=\mathrm{m} \Gamma(\mathrm{m})$ and $\Gamma(1)=1$.

Riemann-Liouville method:

$$
{ }_{a} D_{t}^{\alpha} f(t)=\frac{1}{\Gamma(n-\alpha)}\left(\frac{d}{d t}\right)^{n} \int_{a}^{t} \frac{f(v) d v}{(t-v)^{\alpha-n+1}}
$$

Caputo (1967) method:

$$
{ }_{a}^{c} D_{t}^{\alpha} f(t)=\frac{1}{\Gamma(\alpha-n)} \int_{a}^{t} \frac{f^{(n)}(v) d v}{(t-v)^{\alpha+1-n}}
$$

\subsection{FOD of $f(x)=c x^{0}$}

These three methods for fractional order derivatives are mostly used methods. Due to this case, we will investigate the FOD of each method in this section.

First of all, the results of Euler, Riemann-Liouville and Caputo methods for $\mathrm{f}(\mathrm{x})=\mathrm{cx}^{0}$ are illustrated in Eq.4, Eq.5, and Eq.6. where $\mathrm{c}$ is a constant.

Euler:

$$
\begin{gathered}
\mathrm{n}=1 \text { and } \alpha=\frac{1}{4} \\
\frac{d^{n} x^{m}}{d x^{n}}=\frac{\Gamma(m+1)}{\Gamma(m-n+1)} x^{m-n}=\frac{d^{\frac{1}{4}} x^{0}}{d x^{\frac{1}{4}}}=\frac{\Gamma\left(\frac{5}{4}\right)}{\Gamma\left(\frac{3}{4}\right)} c x^{-\frac{1}{4}}
\end{gathered}
$$

Riemann-Liouville method:

$$
\begin{aligned}
{ }_{a} D_{t}^{\alpha} f(t) & =\frac{1}{\Gamma(n-\alpha)}\left(\frac{d}{d t}\right)^{n} \int_{a}^{t} \frac{f(v) d v}{(t-v)^{\alpha-n+1}} \\
& ={ }_{a} D_{t}^{\frac{2}{3}} f(t)=\frac{1}{\Gamma\left(\frac{1}{3}\right)} \frac{d}{d t} \int_{a}^{t} \frac{c d v}{(t-v)^{\frac{2}{3}}} \\
& =\frac{c}{\Gamma\left(\frac{1}{3}\right)}\left(-\frac{1}{(t-a)^{\frac{2}{3}}}\right) \neq 0
\end{aligned}
$$

The obtained result is inconsistent, since the result is a function of $x$. However, initial function is a constant function and its derivative is zero, since there is no change in the dependent variable.

Caputo method:

$$
\begin{aligned}
{ }_{a}^{C} D_{t}^{\alpha} f(t)= & \frac{1}{\Gamma(\alpha-n)} \int_{a}^{t} \frac{f^{(n)}(v) d v}{(t-v)^{\alpha+1-n}} \\
& =\frac{1}{\Gamma\left(-\frac{1}{3}\right)} \int_{a}^{t} \frac{0 d v}{(t-v)^{\frac{2}{3}+1-1}} \\
& =0
\end{aligned}
$$

The result of Caputo method is consistent. 
The Euler and Riemann-Liouville methods do not work for constant functions as seen in Eq.4 and Eq.5. There is no change in constant function. If there is any change in constant function, it is not a constant function. On contrary, any order derivative of constant function is zero with respect to Caputo method.

\subsection{FOD of $f(x)=x$}

Fractional order derivatives of identity function obtained with respect to Euler, Riemann-Liouville, Caputo methods in this section. Assume that $\mathrm{n}=1$ and $\alpha=\frac{2}{3}$ for all methods.

Euler method:

$$
\frac{d^{\frac{2}{3}} x^{1}}{d x^{\frac{2}{3}}}=\frac{\Gamma(1+1)}{\Gamma\left(1-\frac{2}{3}+1\right)} x^{1-\frac{2}{3}}=\frac{\Gamma(2)}{\Gamma\left(\frac{4}{3}\right)} x^{\frac{1}{3}} \neq 1
$$

Riemann-Liouville method:

$$
\begin{aligned}
{ }_{a} D_{t}^{\alpha} f(t) & =\frac{1}{\Gamma(n-\alpha)}\left(\frac{d}{d t}\right)^{n} \int_{a}^{t} \frac{f(v) d v}{(t-v)^{\alpha-n+1}} \\
& ={ }_{a} D_{t}^{\frac{2}{3}} f(t)=\frac{1}{\Gamma\left(\frac{1}{3}\right)^{t}} \frac{d}{d t} \int_{a}^{t} \frac{x d x}{(t-x)^{\frac{2}{3}-1+1}} \\
& =\frac{1}{\Gamma\left(\frac{1}{3}\right)^{t}} \frac{d}{d t} \int_{a}^{t} \frac{x d x}{(t-x)^{\frac{2}{3}}} \\
& =\frac{1}{\Gamma\left(\frac{1}{3}\right)}\left(3 a(t-a)^{\frac{2}{3}}+\frac{9}{4}(t-a)^{\frac{4}{3}}\right) \neq 1
\end{aligned}
$$

Caputo method:

$$
\begin{aligned}
{ }_{a}^{c} D_{t}^{\alpha} f(t)= & \frac{1}{\Gamma(\alpha-n)} \int_{a}^{t} \frac{f^{(n)}(v) d v}{(t-v)^{\alpha+1-n}} \\
& =\frac{1}{\Gamma\left(-\frac{1}{3}\right)} \int_{a}^{t} \frac{d v}{(t-v)^{\frac{2}{3}+1-1}} \\
& =\frac{1}{\Gamma\left(-\frac{1}{3}\right)}\left(3(t-a)^{\frac{1}{3}}\right) \neq 1
\end{aligned}
$$

It can be seen from definition, the ratio of the change in dependent variable over to the change in independent variable is always 1 (one) for identity function. In this case, the derivative must be 1 in any FOD methods. However, FODs of identity function with respect to Euler, Riemman-Liouville and Caputo methods are different from 1. This means that all methods yielded inconsistent results.
The deficiencies in all three methods for identity function $(\mathrm{f}(\mathrm{x})=\mathrm{x})$ and in two methods (Euler, Riemann-Liouville) for $f(x)=c$ are due to the attention to coefficients and powers of functions during taking derivatives. Another important point in the process of differentiation is that integer coefficients and powers in obtaining formula for differentiation were assumed. After this process, coefficients and powers were assumed as real numbers after a number of specific steps. The formula obtained in this way is not a FOD method; they can be regarded as curve fitting or curve approximation methods.

Euler, Riemann-Liouville and Caputo methods do not work as FOD methods for identity function (Eq.7, Eq.8 and Eq.9).

\section{New Approach for FOD}

The meaning of derivative is the rate of change in the dependent variable versus the changes in the independent variables. At this aim, the derivative of $\mathrm{f}(\mathrm{x})=\mathrm{cx}^{0}$ is that

$$
\lim _{h \rightarrow 0} \frac{f(x+h)-f(x)}{(x+h)-x}=\lim _{h \rightarrow 0} \frac{c-c}{h}=0
$$

In the case of identity function, the derivative is

$$
\lim _{h \rightarrow 0} \frac{f(x+h)-f(x)}{(x+h)-x}=\lim _{h \rightarrow 0} \frac{(x+h)-x}{(x+h)-x}=1
$$

So, the definition for fractional order derivative can be considered as follow.

Definition 1: $\mathrm{f}(\mathrm{x}): \mathrm{R} \rightarrow \mathrm{R}$ is a function, $\alpha \in \mathrm{R}$ and the fractional order derivative can be considered as follows

$$
f^{(\alpha)}(x)=\lim _{h \rightarrow 0} \frac{f^{\alpha}(x+h)-f^{\alpha}(x)}{(x+h)^{\alpha}-x^{\alpha}}
$$

This new approach can be considered for some specific cases. The FOD for $\mathrm{f}(\mathrm{x})=\mathrm{cx}^{0}$

$$
\begin{aligned}
f^{(\alpha)}(x) & =\lim _{h \rightarrow 0} \frac{f^{\alpha}(x+h)-f^{\alpha}(x)}{(x+h)^{\alpha}-x^{\alpha}} \\
& =\lim _{h \rightarrow 0} \frac{c-c}{(x+h)^{\alpha}-x^{\alpha}}=0
\end{aligned}
$$

The FOD for $\mathrm{f}(\mathrm{x})=\mathrm{x}$

$$
\begin{aligned}
f^{(\alpha)}(x) & =\lim _{h \rightarrow 0} \frac{f^{\alpha}(x+h)-f^{\alpha}(x)}{(x+h)^{\alpha}-x^{\alpha}} \\
& =\lim _{h \rightarrow 0} \frac{(x+h)^{\alpha}-x^{\alpha}}{(x+h)^{\alpha}-x^{\alpha}}=1
\end{aligned}
$$

The applications of FODs can be handled by using polynomial, trigonometric, logarithmic and exponential functions. Before handling applications, the new definitions for FOD can be rephrased. The definition 1 is a classical 
definition of derivative, and it has indefinite limit such as $\frac{0}{0}$, while $\mathrm{h}=0$. In this case, Definition 1 can be rephrased as seen in Definition 2.

Definition 2: Assume that $\mathrm{f}(\mathrm{x}): \mathrm{R} \rightarrow \mathrm{R}$ is a function, $\alpha \in \mathrm{R}$ and $\mathrm{L}($.)be a L'Hospital process. The fractional order derivative of $f(x)$ is

$$
\begin{aligned}
& f^{(\alpha)}(x)=\lim _{h \rightarrow 0} L\left(\frac{f^{\alpha}(x+h)-f^{\alpha}(x)}{(x+h)^{\alpha}-x^{\alpha}}\right) \\
& =\lim _{h \rightarrow 0} \frac{\frac{d\left(f^{\alpha}(x+h)-f^{\alpha}(x)\right)}{d h}}{\frac{d\left((x+h)^{\alpha}-x^{\alpha}\right)}{d h}}
\end{aligned}
$$

\section{Applications of New Approach for FOD}

The applications of FOD (F.O.D.) for logarithmic, trigonometric, exponential and polynomial functions were done in this section. The FOD of $\sin (x)$ is $f^{(\alpha)}(x)=\frac{\cos (x)(\sin (x))^{\alpha-1}}{x^{\alpha-1}}$. The graphics of FOD for $\alpha=0.5, \alpha=1, \alpha=1.5, \alpha=2, \alpha=2.5$ and function itself are seen in Figure 1.
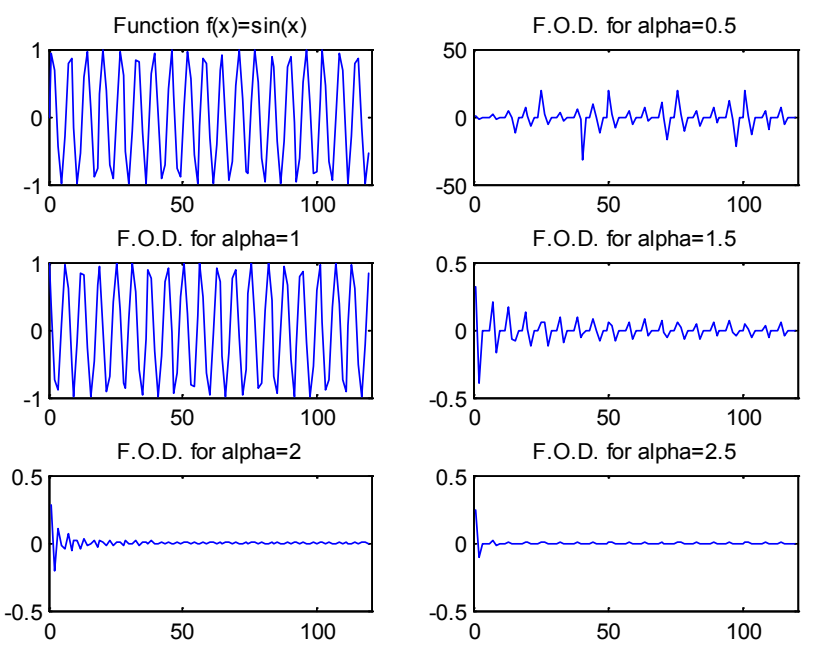

Figure 1. Graphics of FOD for $\alpha=0.5, \alpha=1, \alpha=1.5, \alpha=2, \alpha=2.5$ and function itself.

The FOD of $\cos (\mathrm{x})$ is $f^{(\alpha)}(x)=\frac{-\sin (x)(\cos (x))^{\alpha-1}}{x^{\alpha-1}}$. The graphics of FOD for $\alpha=0.5, \alpha=1, \alpha=1.5, \alpha=2, \alpha=2.5$ and function itself are seen in Figure 2. The FOD of $\tan (\mathrm{x})$ is $f^{(\alpha)}(x)=\frac{\left(1+\tan ^{2}(x)\right)(\tan (x))^{\alpha-1}}{x^{\alpha-1}}$. The graphics of FOD for $\alpha=0.5, \alpha=1, \alpha=1.5, \alpha=2, \alpha=2.5$ and function itself are seen in Figure 3.
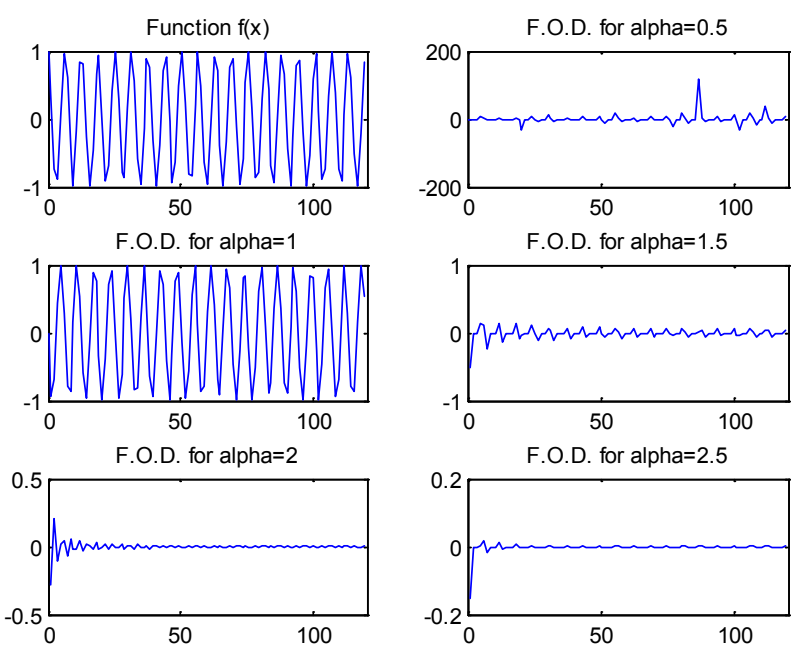

Figure 2. Graphics of FOD for $\alpha=0.5, \alpha=1, \alpha=1.5, \alpha=2, \alpha=2.5$ and function itself $(\mathrm{f}(\mathrm{x})=\cos (\mathrm{x}))$.
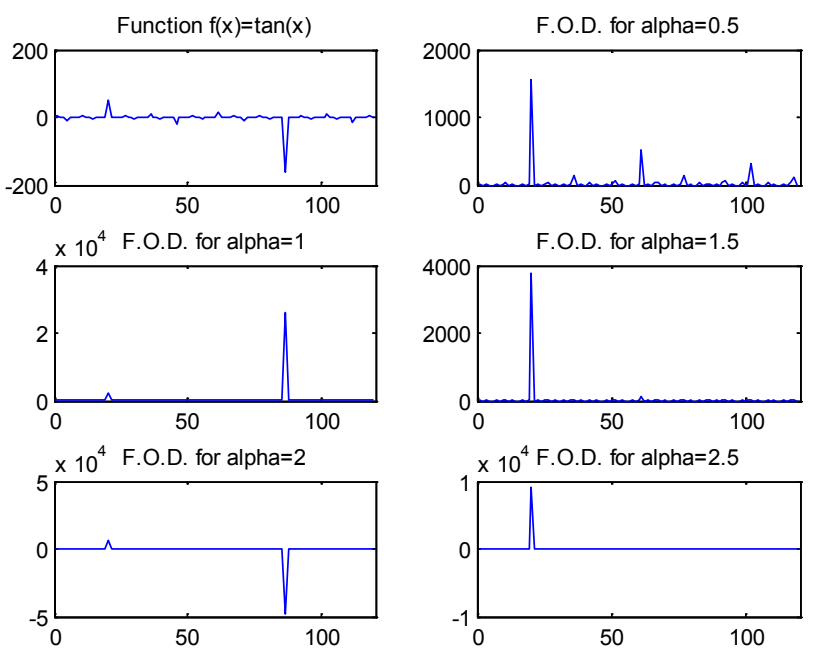

Figure 3. Graphics of FOD for $\alpha=0.5, \alpha=1, \alpha=1.5, \alpha=2, \alpha=2.5$ and function itself $(\mathrm{f}(\mathrm{x})=\tan (\mathrm{x}))$.
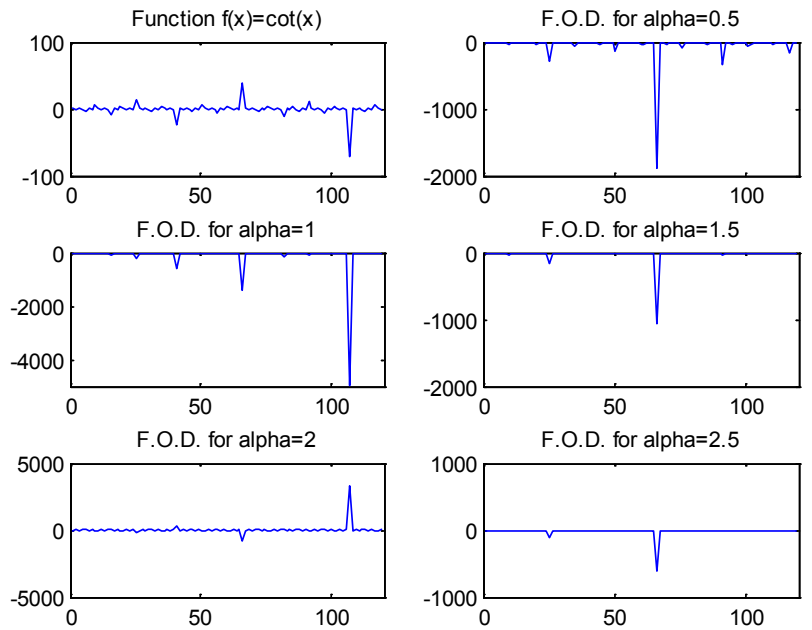

Figure 4. Graphics of FOD for $\alpha=0.5, \alpha=1, \alpha=1.5, \alpha=2, \alpha=2.5$ and function itself $(f(x)=\cot (x))$. 
The FOD of $\cot (\mathrm{x})$ is $f^{(\alpha)}(x)=\frac{-\left(1+\cot ^{2}(x)\right)(\cot (x))^{\alpha-1}}{x^{\alpha-1}}$. The graphics of FOD for $\alpha=0.5, \alpha=1, \alpha=1.5, \alpha=2, \alpha=2.5$ and function itself are seen in Figure 4. The FOD of $f(x)=x^{4}-5 x^{3}+x-2$ is

$f^{(\alpha)}(x)=\frac{\left(4 x^{3}-15 x^{2}+1\right)\left(x^{4}-5 x^{3}+x-2\right)^{\alpha-1}}{x^{\alpha-1}}$. The graphics of FOD for $\alpha=0.5, \alpha=1, \alpha=1.5, \alpha=2, \alpha=2.5$ and function itself are seen in Figure 5.
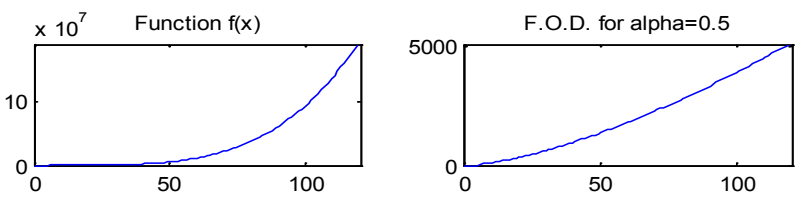

$\times 10^{6}$ F.O.D. for alpha $=1$
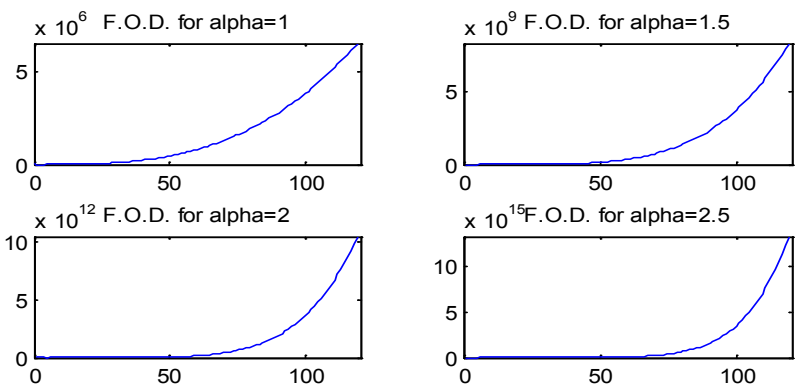

Figure 5. Graphics of FOD for $\alpha=0.5, \alpha=1, \alpha=1.5, \alpha=2, \alpha=2.5$ and function itself $\left(f(x)==x^{4}-5 x^{3}+x-2\right)$.

The FOD of $f(x)=\frac{1}{x^{4}-5 x^{3}+x-2}$ is

$$
f^{(\alpha)}(x)=\frac{\left(-\frac{4 x^{3}-15 x^{2}+1}{\left(x^{4}-5 x^{3}+x-2\right)^{2}}\right)\left(\frac{1}{x^{4}-5 x^{3}+x-2}\right)^{\alpha-1}}{x^{\alpha-1}} .
$$

The graphics of FOD for $\alpha=0.5, \alpha=1, \alpha=1.5, \alpha=2, \alpha=2.5$ and function itself are seen in Figure 6.
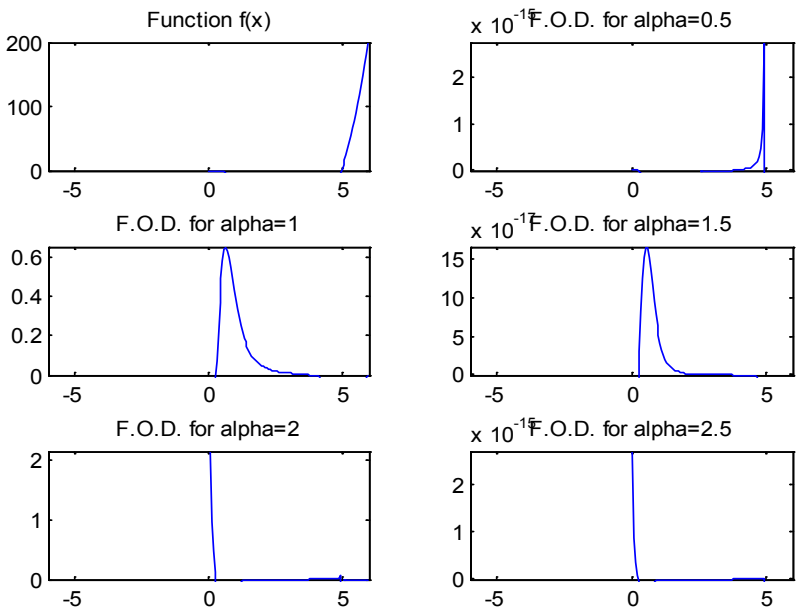

Figure 6. Graphics of FOD for $\alpha=0.5, \alpha=1, \alpha=1.5, \alpha=2, \alpha=2.5$ and function itself $\left(f(x)=\frac{1}{x^{4}-5 x^{3}+x-2}\right)$.
The FOD of $\mathrm{f}(\mathrm{x})=\ln (\mathrm{x})$ is $f^{(\alpha)}(x)=\frac{\left(\frac{1}{x}\right)(\ln (x))^{\alpha-1}}{x^{\alpha-1}}$. The graphics of FOD for $\alpha=0.5, \alpha=1, \alpha=1.5, \alpha=2, \alpha=2.5$ and function itself are seen in Figure 7. The FOD of $f(x)=e^{x}$ is $f^{(\alpha)}(x)=\frac{e^{x}\left(e^{x}\right)^{\alpha-1}}{x^{\alpha-1}}$. The graphics of FOD for $\alpha=0.5$, $\alpha=1, \alpha=1.5, \alpha=2, \alpha=2.5$ and function itself are seen in Figure 8. The FOD of $\mathrm{f}(\mathrm{x})=2^{\mathrm{x}}$ is $f^{(\alpha)}(x)=\frac{2^{x} \ln (2)\left(2^{x}\right)^{\alpha-1}}{x^{\alpha-1}}$. The graphics of FOD for $\alpha=0.5, \alpha=1, \alpha=1.5, \alpha=2, \alpha=2.5$ and function itself are seen in Figure 9.
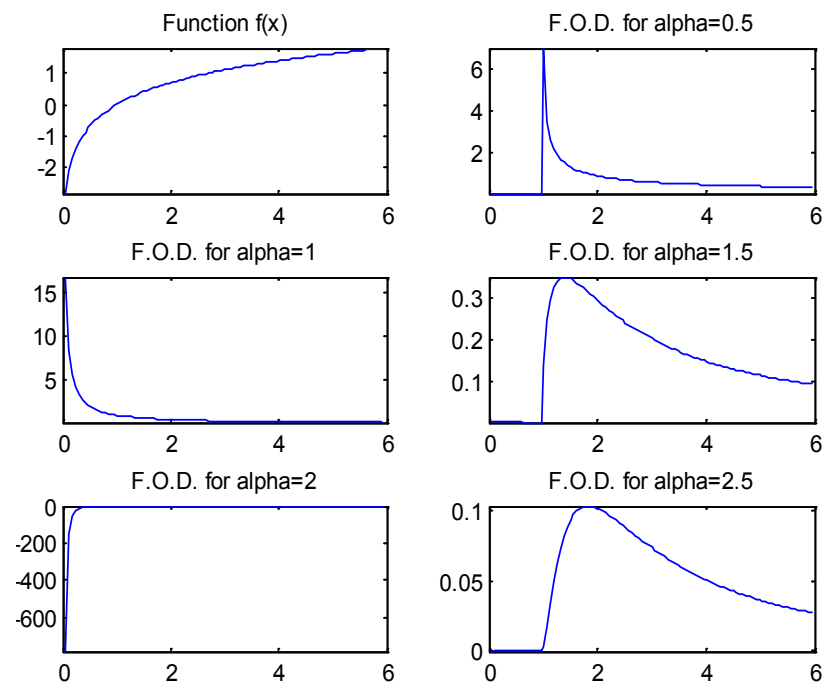

Figure 7. Graphics of FOD for $\alpha=0.5, \alpha=1, \alpha=1.5, \alpha=2, \alpha=2.5$ and function itself $(\mathrm{f}(\mathrm{x})=\ln (\mathrm{x}))$.
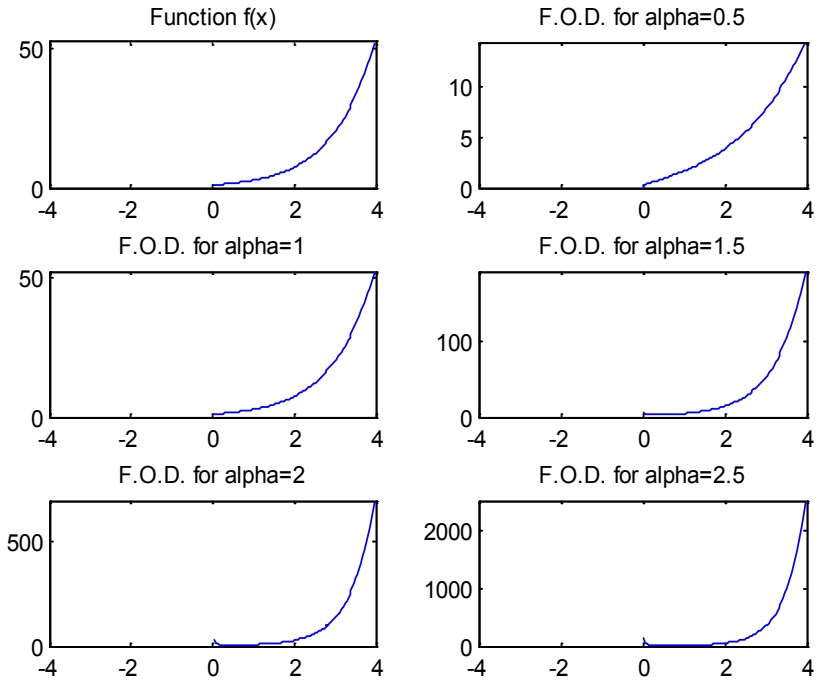

Figure 8. Graphics of FOD for $\alpha=0.5, \alpha=1, \alpha=1.5, \alpha=2, \alpha=2.5$ and function itself $\left(f(x)=e^{x}\right)$. 

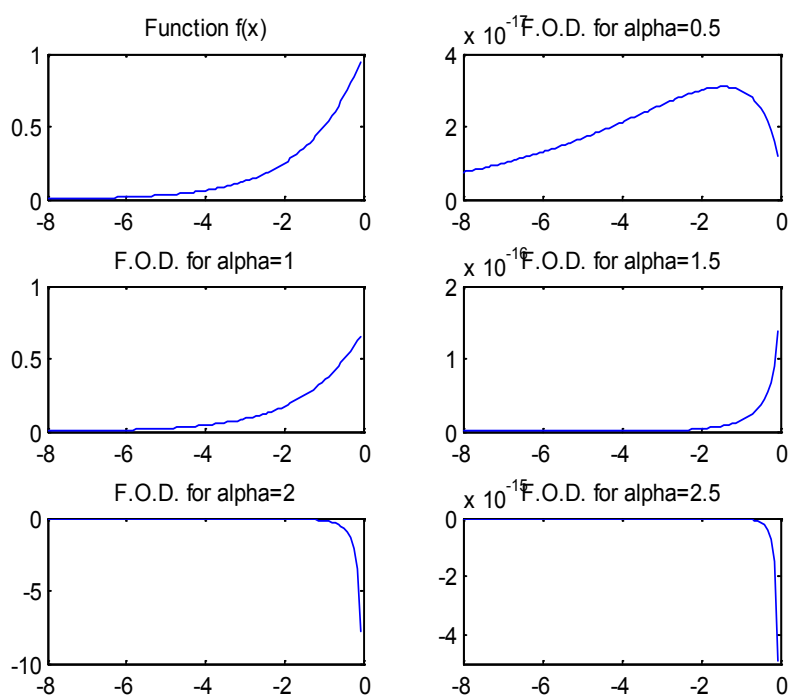

Figure 9. Graphics of FOD for $\alpha=0.5, \alpha=1, \alpha=1.5, \alpha=2, \alpha=2.5$ and function itself $\left(f(x)=2^{x}\right)$.

\section{FODs of Some Functions}

The FODs of exponential, logarithmic, trigonometric and polynomial functions will be obtained in this section.

There is a relationship with classical derivative and fractional order derivative. This case is seen in the following cases. If the order of fractional order derivative is $\alpha$, then the fractional order derivative of any function is

$$
f^{(\alpha)}(x)=\left(\frac{f(x)}{x}\right)^{\alpha-1} f^{\prime}(x)
$$

Assume that $\mathrm{f}(\mathrm{x})$ is a function and $\mathrm{f}=\sin \mathrm{x}$ and assume that the order of fractional order derivative is $\alpha$.

\begin{tabular}{|c|c|c|}
\hline$\alpha$ & $f(x)$ & $f^{(\alpha)}(x)=\left(\frac{\sin x}{x}\right)^{\alpha-1} \cos x$ \\
\hline$\frac{1}{2}$ & $\sin x$ & $f^{\left(\frac{1}{2}\right)}(x)=\cos x \sqrt{\frac{x}{\sin x}}$ \\
\hline 2 & $\sin x$ & $f^{(2)}(x)=\frac{\sin x \cos x}{x}$ \\
\hline$-\frac{1}{2}$ & $\sin x$ & $f^{\left(-\frac{1}{2}\right)}(x)=\cos x \sqrt{\frac{x^{3}}{\sin ^{3} x}}$ \\
\hline-2 & $\sin x$ & $f^{(-2)}(x)=\cos x \frac{x^{3}}{\sin ^{3} x}$ \\
\hline
\end{tabular}

Assume that $\mathrm{f}(\mathrm{x})$ is a function and $\mathrm{f}=\cos \mathrm{x}$ and assume that the order of fractional order derivative is $\alpha$.

\begin{tabular}{|c|c|c|}
\hline$\alpha$ & $\mathrm{f}(\mathrm{x})$ & $f^{(\alpha)}(x)=-\sin x\left(\frac{\cos x}{x}\right)^{\alpha-1}$ \\
\hline$\frac{1}{2}$ & $\cos x$ & $f^{\left(\frac{1}{2}\right)}(x)=-\sin \sqrt{\frac{x}{\cos x}}$ \\
\hline 2 & $\operatorname{cosx}$ & $f^{(2)}(x)=-\frac{\sin x \cos x}{x}$ \\
\hline$-\frac{1}{2}$ & $\cos x$ & $f^{\left(-\frac{1}{2}\right)}(x)=-\sin x \sqrt{\frac{x^{3}}{\cos ^{3} x}}$ \\
\hline-2 & $\operatorname{cosx}$ & $f^{(-2)}(x)=\cos x \frac{x^{3}}{\sin ^{3} x}$ \\
\hline
\end{tabular}

Assume that $\mathrm{f}(\mathrm{x})$ is a function and $\mathrm{f}=\tan \mathrm{x}$ and assume that the order of fractional order derivative is $\alpha$.

\begin{tabular}{|c|c|c|}
\hline$\alpha$ & $\mathrm{f}(\mathrm{x})$ & $f^{(\alpha)}(x)=\left(1+\tan ^{2} x\right)\left(\frac{\tan x}{x}\right)^{\alpha-1}$ \\
\hline$\frac{1}{2}$ & $\tan \mathrm{f}$ & $f^{\left(\frac{1}{2}\right)}(x)=\left(1+\tan ^{2} x\right) \sqrt{\frac{x}{\tan x}}$ \\
\hline 2 & $\tan \mathrm{f}$ & $f^{(2)}(x)=\left(1+\tan ^{2} x\right) \frac{\tan x}{x}$ \\
\hline$-\frac{1}{2}$ & $\tan \mathrm{f}$ & $f^{\left(-\frac{1}{2}\right)}(x)=\left(1+\tan ^{2} x\right) \sqrt{\frac{x^{3}}{\tan ^{3} x}}$ \\
\hline-2 & $\tan x$ & $f^{(-2)}(x)=\left(1+\tan ^{2} x\right) \frac{x^{3}}{\tan ^{3} x}$ \\
\hline
\end{tabular}

Assume that $\mathrm{f}(\mathrm{x})$ is a function and $\mathrm{f}=\cot \mathrm{x}$ and assume that the order of fractional order derivative is $\alpha$.

\begin{tabular}{|c|c|c|}
\hline$\alpha$ & $f(x)$ & $f^{(\alpha)}(x)=-\left(1+\cot ^{2} x\right)\left(\frac{\cot x}{x}\right)^{\alpha-1}$ \\
\hline$\frac{1}{2}$ & $\cot x$ & $f^{\left(\frac{1}{2}\right)}(x)=-\left(1+\cot ^{2} x\right) \sqrt{\frac{x}{\cot x}}$ \\
\hline 2 & $\cot x$ & $f^{(2)}(x)=-\left(1+\cot ^{2} x\right) \frac{\cot x}{x}$ \\
\hline$-\frac{1}{2}$ & $\cot x$ & $f^{\left(-\frac{1}{2}\right)}(x)=-\left(1+\cot ^{2} x\right) \sqrt{\frac{x^{3}}{\cot ^{3} x}}$ \\
\hline-2 & $\cot x$ & $f^{(-2)}(x)=-\left(1+\cot ^{2} x\right) \frac{x^{3}}{\cot ^{3} x}$ \\
\hline
\end{tabular}


Assume that $\mathrm{f}(\mathrm{x})$ is a function and $\mathrm{f}=\ln \mathrm{x}$ and assume that the order of fractional order derivative is $\alpha$.

\begin{tabular}{|c|c|c|}
\hline$\alpha$ & $f(x)$ & $f^{(\alpha)}(x)=\frac{\ln ^{\alpha-1} x}{x^{\alpha}}$ \\
\hline$\frac{1}{2}$ & $\ln x$ & $f^{\left(\frac{1}{2}\right)}(x)=\frac{1}{\sqrt{x \ln x}}$ \\
\hline 2 & $\ln x$ & $f^{(2)}(x)=\frac{\ln x}{x^{2}}$ \\
\hline$-\frac{1}{2}$ & $\ln x$ & $f^{\left(-\frac{1}{2}\right)}(x)=\frac{1}{\sqrt{x \ln ^{3} x}}$ \\
\hline-2 & $\ln x$ & $f^{(-2)}(x)=\frac{x^{2}}{\ln ^{3} x}$ \\
\hline
\end{tabular}

Assume that $\mathrm{f}(\mathrm{x})$ is a function and $\mathrm{f}=\mathrm{x}^{\mathrm{n}}$ and assume that the order of fractional order derivative is $\alpha$.

\begin{tabular}{|c|c|c|}
\hline$\alpha$ & $\mathrm{f}(\mathrm{x})$ & $f^{(\alpha)}(x)=n x^{\alpha(n-1)}$ \\
\hline$\frac{1}{2}$ & $\mathrm{x}^{\mathrm{n}}$ & $f^{\left(\frac{1}{2}\right)}(x)=n \sqrt{x^{n-1}}$ \\
\hline 2 & $\mathrm{x}^{\mathrm{n}}$ & $f^{(2)}(x)=n x^{2(n-1)}$ \\
\hline$-\frac{1}{2}$ & $\mathrm{x}^{\mathrm{n}}$ & $f^{\left(-\frac{1}{2}\right)}(x)=\frac{n}{\sqrt{x^{n-1}}}$ \\
\hline-2 & $\mathrm{x}^{\mathrm{n}}$ & $f^{(-2)}(x)=\frac{n}{x^{2(n-1)}}$ \\
\hline
\end{tabular}

Assume that $\mathrm{f}(\mathrm{x})$ is a function and $\mathrm{f}=\mathrm{e}^{\mathrm{ax}}$ and assume that the order of fractional order derivative is $\alpha$.

\begin{tabular}{|c|c|c|}
\hline$\alpha$ & $\mathrm{f}(\mathrm{x})$ & $f^{(\alpha)}(x)=\left(\frac{e^{a x}}{x}\right)^{\alpha-1} a e^{a x}$ \\
\hline$\frac{1}{2}$ & $\mathrm{e}^{\mathrm{ax}}$ & $f^{\left(\frac{1}{2}\right)}(x)=a \sqrt{x e^{a x}}$ \\
\hline 2 & $\mathrm{e}^{\mathrm{ax}}$ & $f^{(2)}(x)=\frac{a}{x} e^{2 a x}$ \\
\hline$-\frac{1}{2}$ & $\mathrm{e}^{\mathrm{ax}}$ & $f^{\left(-\frac{1}{2}\right)}(x)=a \sqrt{\frac{x^{3}}{e^{a x}}}$ \\
\hline-2 & $\mathrm{e}^{\mathrm{ax}}$ & $f^{(-2)}(x)=a \frac{x^{3}}{e^{2 a x}}$ \\
\hline
\end{tabular}

Assume that $\mathrm{f}(\mathrm{x})$ is a function and $\mathrm{f}=\sin ^{\mathrm{n}} \mathrm{x}$ and assume that the order of fractional order derivative is $\alpha$.

\begin{tabular}{|c|c|c|}
\hline$\alpha$ & $\mathrm{f}(\mathrm{x})$ & $f^{(\alpha)}(x)=\frac{n \cos x \sin ^{\alpha n-1} x}{x^{\alpha-1}}$ \\
\hline$\frac{1}{2}$ & $\sin ^{\mathrm{n} x}$ & $f^{\left(\frac{1}{2}\right)}(x)=n \tan x \sqrt{x \sin ^{n} x}$ \\
\hline 2 & $\sin ^{\mathrm{n} x}$ & $f^{(2)}(x)=\frac{n \tan x \sin ^{2 n} x}{x}$ \\
\hline$-\frac{1}{2}$ & $\sin ^{\mathrm{n} x}$ & $f^{\left(-\frac{1}{2}\right)}(x)=n \cos x \sqrt{\frac{x^{3}}{\sin ^{n+2} x}}$ \\
\hline-2 & $\sin ^{\mathrm{n} x}$ & $f^{(-2)}(x)=n \cos x \frac{x^{3}}{\sin ^{2 n+1} x}$ \\
\hline
\end{tabular}

\section{Conclusions}

The FOD methods in the literature have some important deficiencies, since they assumed the order of derivation is integer up to a specific derivation step. This caused involvement of deficiencies in the obtained formula. FOD was redefined in this paper. The applications of FOD in this paper are real parts of FOD, since the results of FOD are functions of complex variables or complex numbers. Continuity was obtained by serial expansion of any function in the methods used in the literature. This case also caused deficiencies or errors.

Assume that $\alpha=\frac{\beta}{\delta}$ and $\delta \neq 0$. The FOD of $\mathrm{f}(\mathrm{x})$ is

$$
\begin{aligned}
f^{(\alpha)} & =\frac{f^{\prime}(x) f^{\alpha-1}(x)}{x^{\alpha-1}} \\
& =f^{\prime}(x) \sqrt[\delta]{\frac{f^{\beta-\delta}(x)}{x^{\beta-\delta}}} \\
& =f^{\prime}(x) \sqrt[\delta]{\left(\frac{f(x)}{x}\right)^{\beta-\delta}}
\end{aligned}
$$

If the FOD is a function of complex variables, then $\mathrm{f}^{(\alpha)}(\mathrm{x})=\mathrm{g}(\mathrm{x})+\mathrm{ih}(\mathrm{x})$ where $\mathrm{i}$ is the $i=\sqrt{-1}$.

If $\mathrm{f}(\mathrm{x})<0$, there will be two cases:

Case 1: Assume that $\delta$ is odd.

$$
\text { If }\left(\frac{f(x)}{x}\right)^{\beta-\delta} \geq 0 \text { or }\left(\frac{f(x)}{x}\right)^{\beta-\delta}<0,
$$

then the obtained function $\mathrm{f}^{(\alpha)}(\mathrm{x})$ is a real function and $h(x)=0$ for both cases. Since the multiplication of any negative number in odd steps yields a negative number.

Case 2: Assume that $\delta$ is even.

If $\left(\frac{f(x)}{x}\right)^{\beta-\delta} \geq 0$,

then $h(x)=0$ and $f^{(\alpha)}(x)$ is a real function. 
If $\left(\frac{f(x)}{x}\right)^{\beta-\delta}<0$,

then the multiplication of any number in even steps yields a positive number for real numbers. However, it yields a negative result for complex numbers, $\mathrm{so}, \mathrm{h}(\mathrm{x}) \neq 0$. This means that $f^{(\alpha)}(x)$ is a complex function.

In fact, $f^{(\alpha)}(x)$ is a complex function for both cases. The $h(x)=0$ for some situations.

\section{REFERENCES}

[1] S. Pooseh, R. Almeida, D.F.M. Torres, Discrete direct methods in the fractional calculus of variations, Computers and Mathematics with Applications, doi:10.1016/j.camwa.2013.01.045.

[2] S.P.Mirevski, L.Boyadjiev, R.Scherer, On the Riemann-Liouville Fractional Calculus, g-Jacobi Functions and F.Gauss Functions, Applied Mathematics and Computation, 187; 315-325 (2007).

[3] S.E.Schiavone, W.Lamb, A Fractional Power Approach to Fractional Calculus, Journal of Mathematical Analysis and
Applications, 149; 377-401 (1990).

[4] A.S. Bataineh, A.K. Alomari, M.S.M. Noorani, I. Hashim, R. Nazar, Series Solutions of Systems of Nonlinear Fractional Differential Equations, Acta Applied Mathematics, 105; 189-198 (2009).

[5] K.Diethelm, N.J. Ford, A.D. Freed, Yu Luchko, Algorithms fort he Fractional Calculus: A Selection of Numerical Methdos, Computer Methods in Applied Mechanics and Engineering, 194; 743-773 (2005).

[6] C.Li, A.Chen, J.Ye, Numerical Approaches to Fractional Calculus and Fractional Ordinary Differential Equation, Journal of Computational Physics, 230; 3352-3368 (2011).

[7] C.Li, D.Qian, Y.-Q. Chen, On Riemann-Liouville and Caputo Derivatives, Discrete Dynamics in Nature and Society, 2011, DOI: $10.1155 / 2011 / 562494$.

[8] M.Ö. Efe, Fractional Order Sliding Mode Control with Reaching Law Approach, Turkish Journal of Electrical Engineering \& Computer Science, 18; $731-747$ (2010).

[9] A. Karcı, Kesirli Türev için Yapılan Tanımlamaların Eksiklikleri ve Yeni Yaklașım, TOK-2013 Turkish Automatic Control National Meeting and Exhibition. 